\title{
Riesgo de hemorragia postquirúrgica en pacientes bajo tratamiento antitrombótico sometidos a cirugía oral: Revisión Sistemática y Metaanálisis.
}

\author{
Risk of postsurgical hemorrhage in patients with \\ antitrombotic treatment undergoing oral surgery: \\ A systematic review and Metanalysis.
}

\author{
Julio Villanueva*1,2,3, Diego Vergara ${ }^{1}$, Lorena Núñez ${ }^{1,2}$, Gabriel Zamorano ${ }^{1,2}$, Sebastián Zapata ${ }^{1,2}$, Josefina \\ Salazar ${ }^{1}$, Ana Alarcon ${ }^{1,2,3}$, Rosa Rojo ${ }^{4}$, Stefan Domancic ${ }^{1,2}$, Nicolás Yanine ${ }^{1,2,3}$, Ignacio Araya ${ }^{1,2}$
}

\author{
1 Departamento de Cirugía y Traumatología Bucal y \\ Maxilofacial, Facultad de Odontología, Universidad \\ de Chile, Santiago, Chile. \\ 2 Unidad de Odontología Basada en Evidencia, \\ Centro Cochrane, Santiago, Chile \\ 3 Unidad de Cirugía Maxilofacial, Hospital Clínico \\ San Borja Arriaran. Santiago, Chile \\ 4 Universidad Rey Juan Carlos, Madrid, España. \\ ${ }^{*}$ Correspondencia autor: Julio Villanueva | \\ Dirección: Sergio Livingstone P 943 Independencia \\ (8380492). Santiago de Chile. | Teléfono \\ +56229781841| Email: javm@uchile.cl \\ Trabajo recibido el 27/11/2017. Aprobado para su \\ publicación el 15/04/2018
}

\section{RESUMEN}

Introducción: el objetivo de esta revisión fue determinar el riesgo de hemorragia postoperatoria en pacientes con tratamiento anticoagulante oral (TACO) sometidos a cirugía oral que no suspenden su tratamiento comparado con quienes lo modifican o suspenden. Materiales y métodos: se realizó una búsqueda en CENTRAL, Medline y EMBASE, junto con una revisión manual de revistas especializadas y resúmenes de la IADR. Dos revisores realizaron la selección de estudios, evaluación de riesgo de sesgo y extracción de datos de forma independiente. Se seleccionaron los ensayos clínicos aleatorizados que miden la aparición de hemorragias en pacientes sometidos a procedimientos quirúrgicos orales con TACO en comparación con un grupo que interrumpió o modificó su terapia. Resultados: Solo 5 estudios cumplieron los criterios de inclusión (549 pacientes). El metanálisis mostró que el mantenimiento de la TACO no aumenta el riesgo de hemorragia postoperatoria (riesgo relativo: 1,41 [0,93 - 2,16], IC del $95 \% p=0,11$ ) en comparación con la interrupción del tratamiento. Conclusión: Aunque se encontró una mayor cantidad de hemorragia postoperatoria en pacientes con TACO comparado con quienes lo interrumpieron o modificaron, esta diferencia no fue estadística ni clínicamente significativa. Por lo tanto, TACO no debe suspenderse en pacientes sometidos a cirugía oral.

PALABRAS CLAVE

Cirugía oral, Hemorragia, Anticoagulantes orales.

Rev. Clin. Periodoncia Implantol. Rehabil. Oral Vol. 11(2); 121-127, 2018.

\begin{abstract}
Introduction: This systematic review aims to determine the postoperative bleeding risk in patients on oral anticoagulant therapy (OAT) undergoing oral surgical procedures when continuing with the treatment, compared with those modifying or discontinuing the treatment. Materials and methods: A search was performed using CENTRAL,Medline and EMBASE, in conjunction with a manual review of indexes of specialized journals and abstracts of the IADR. Study selection, assessments of risk of bias and data extraction were performed independently by 2 reviewers. Randomized clinical trials measuring the occurrence of bleeding in patients on OAT undergoing oral surgical procedures compared with a group discontinuing or modifying their therapy were selected. Results: A total of 5 studies were included based on inclusion criteria (549 patients). The metaanalysis showed that the maintenance of OAT does not increasethe risk of postoperative bleeding (relative risk [RR] $1.41[0.93-2.16] ; 95 \% \mathrm{Cl} p=0,11$ )compared with the discontinuation of therapy. Conclusion: Although a larger quantityof oral postoperative bleeding episodes were found in patients continuing with OATcompared with patients discontinuing or modifying their therapy, this difference was neither statistically nor clinically significant. Therefore, OAT should not be discontinued in patients undergoing oral surgery.

KEYWORDS
\end{abstract}

Oral surgical procedures, Bleeding, Anticoagulants.

Rev. Clin. Periodoncia Implantol. Rehabil. Oral Vol. 11(2); 121-127, 2018. 


\section{INTRODUCCIÓN}

Actualmente el tratamiento farmacológico anticoagulante es utilizado cada vez con mayor frecuencia, debido al aumento de la expectativa de vida y de la incidencia de enfermedades cardiovasculares asociadas al desarrollo de tromboembolismo ${ }^{(1)}$, siendo utilizada para el tratamiento de trombosis venosa profunda, embolismo pulmonar, como coadyuvante en el tratamiento de enfermedades valvulares con sustitutos prostéticos y en la fibrilación auricular crónica ${ }^{(2)}$, como también, relacionada a esta última, en los accidentes vasculares encefálicos de tipo trombótico(3). Dentro de los anticoagulantes más utilizados y administrados por vía oral, se encuentran antagonistas de la Vitamina K como el Acenocumarol y la Warfarina ${ }^{(4,5)}$. El manejo de dosis de este medicamento es complejo, debiendo adaptarse su uso a cada paciente, dado que su acción puede durar entre 2 y 5 días, requiriendo entonces de una monitorización constante.

La necesidad de tratamiento odontológico en relación a la cirugía oral constituye un constante dilema para el manejo de estos pacientes bajo la terapia anticoagulante oral (TACO), debido a que no existe un estricto consenso respecto a la conducta clínica ${ }^{(6)}$, existiendo una corriente que asume un elevado riesgo hemorrágico, suspendiendo habitualmente la terapia un par de días previo a la intervención y retomándola un día después, en general en acuerdo con el médico tratante, versus otra corriente que mantiene la terapia dentro de los valores objetivos, previo control con exámenes de laboratorio, con el fin de evitar el riesgo tromboembólico que supone la suspensión de fármaco ${ }^{(1,7-9)}$. En general, la controversia está sustentada en la capacidad de controlar una hemorragia con medidas hemostáticas locales durante los procedimientos de cirugía oral( ${ }^{(10)}$ continuando el TACO, dado que estos pacientes mantienen su capacidad hemostática funcional, pero disminuida ${ }^{(11)}$; versus el otro punto de vista, donde algunos tratantes prefieren eliminar la influencia del medicamento y con ello, la posibilidad de hemorragia, asumiendo el mayor riesgo tromboembólico por el cual éste fue indicado. Es importante considerar que el TACO ha demostrado reducir la probabilidad de aparición de primeros eventos tromboembólicos en alrededor de un 70\% y en aproximadamente $90 \%$ las recurrencias de los mismos ${ }^{(12,13)}$.

Importantes entidades como la American Heart Association han realizado recomendaciones al respecto en su guía para el manejo de la enfermedad valvular cardíaca el $2014^{(14)}$, sin embargo, y a pesar de la recomendación de no suspender el TACO, aún existe mucha disparidad de criterio entre los dentistas alrededor del mundo frente a procedimientos de cirugía oral en pacientes anticoagulados, describiendo variadas conductas clínicas en algunos estudios, donde lo han cuantificado mediante encuestas de la conducta terapéutica en estas situaciones clínicas, existiendo alrededor de un $8 \%$ de profesionales que prefiere simplemente no tratar a estos pacientes y de aquellos que sí lo hacen, entre un 20 a un $25 \%$ no chequea el INR (International Normalized Ratio), indicador que evalúa de forma estandarizada el estado de coagulación/anti-coagulación de un paciente haciendo una relación entre su tiempo de protrombina y el de un control normal, antes de realizar procedimientos quirúrgicos y de los que sí lo hacen, alrededor de un 17 a un $21 \%$ lo lleva a cabo hasta 72 horas antes del mismo(15,16). Lo descrito previamente puede llegar a constituir un problema, no solo de decisión clínica, sino en cuanto a lo oportuna y correcta intervención, comprometiendo la seguridad del paciente, con los consiguientes aspectos médico legales.

En la literatura existen estudios con resultados disímiles respecto al riesgo hemorrágico y la suspensión de la TACO en pacientes sometidos a cirugía oral. Adicionalmente, no se ha logrado consenso, existiendo una disparidad de criterios clínicos e indicaciones, incluso en una revisión sistemática realizada por Nematullah en $2009^{(1)}$, en donde mediante un análisis de los estudios se observó una ligera tendencia a favor de la suspensión, aunque sin mediar un análisis estadístico de por medio, dada la inclusión de estudios de diferente naturaleza. Lo anterior hace necesaria la realización de un estudio que incluya la nueva evidencia disponible y que, en el mejor de los casos, mediante un tratamiento de los datos apropiado, se logre resolver esta controversia.

El objetivo de esta revisión sistemática es determinar el riesgo de hemorragia posquirúrgica en pacientes con TACO sometidos a procedimientos de cirugía oral al continuar el tratamiento en comparación a aquellos que lo modifican o suspenden.

\section{METODOLOGÍA}

Se llevó a cabo una revisión sistemática de ensayos clínicos aleatorizados teniendo en consideración las recomendaciones de la declaración PRISMA para el reporte de revisiones sistemáticas ${ }^{(17)}$. Los ensayos clínicos elegidos midieron la ocurrencia de eventos hemorrágicos en pacientes bajo TACO sometidos a diferentes procedimientos de cirugía oral.

\section{Métodos de búsqueda para identificación de los estudios}

Se realizó la búsqueda electrónica en marzo del 2017, en las siguientes bases de datos: CENTRAL, Medline vía PubMed y EMBASE (entre los años 1964-junio 2017), para identificar los ensayos clínicos aleatorizados pertinentes. En la Tabla 1 se encuentran la estrategia de búsqueda, los términos $\mathrm{MeSH}$ y palabras claves utilizadas en las búsquedas.

Tabla 1. Estrategias de Búsqueda.

\section{A) ESTRATEGIA DE BUSQUEDA EN PUBMED}

\section{8 hints}

((((((((“Surgery, Oral”[Mesh] OR “Oral Surgical Procedures”[Mesh]))

OR $((((()((($ oral surgery[Title/Abstract]) OR extraction[Title/Abstract])

OR third molar[Title/Abstract])

OR periapical surgery[Title/Abstract]) OR frenectomy[Title/

Abstract]) OR fenestration) OR dental implant*[Title/Abstract]))

AND ((dental[Title/Abstract]) OR oral[Title/Abstract])))))) AND

((((“Anticoagulants” [Pharmacological Action] OR

\section{"Anticoagulants"[Mesh]) OR ("Heparinoids"[Mesh] OR}

“4-Hydroxycoumarins"[Mesh] $)))$ OR $((()((()((()((()(((($ acenocoumarol[ Title/Abstract]) OR warfarin[Title/Abstract]) OR heparin[Title/Abstract]) OR rivaroxaban[Title/Abstract]) OR dabigatran[Title/Abstract])

OR apixaban[Title/Abstract]) OR vitamin $\mathrm{K}$ antagonist[Title/Abstract]) OR

atromentin[Title/Abstract]) OR brodifacoum[Title/Abstract]) OR phenindione[Title/Abstract]) OR phenprocoumon[Title/Abstract]) OR fondaparinux[Title/Abstract]) OR idraparinux[Title/Abstract]) OR

edoxaban[Title/Abstract]) OR betrixaban[Title/Abstract]) OR

darexaban[Title/Abstract]) OR hirudin[Title/Abstract]) OR lepirudin[Title/Abstract]) OR bivalirudin[Title/Abstract]) OR argatroban[Title/Abstract]) OR ximelagatran[Title/Abstract]))))) AND $((()((()(($ randomized controlled trial [pt]) OR

controlled clinical trial [pt]) OR randomized [tiab]) OR placebo [tiab]) OR drug therapy [sh]) OR randomly [tiab]) OR trial [tiab]) OR groups [tiab])) NOT ((animals [mh] NOT humans [mh]))))

\section{B) ESTRATEGIA DE BUSQUEDA EN EMBASE} 46 hints

'oral surgery'/exp OR 'oral surgery' AND ('anticoagulant agent'/exp OR 'anticoagulant agent' OR 'heparinoid'/exp OR 'heparinoid' OR 'coumarin derivative'/exp OR 'coumarin derivative') AND ([systematic review]/lim OR [controlled clinical trial]/lim OR

[randomized controlled trial]/lim OR [meta analysis]/lim) AND [humans]/lim AND

[embase]/lim NOT [15-3-2015]/sd

En junio del 2017 se realizó la búsqueda manual en los índices de las siguientes revistas de la especialidad:

- Journal of Oral and Maxillofacial Surgery

- International Journal of Oral and Maxillofacial Surgery

- British Journal of Oral and Maxillofacial Surgery

- Journal of the American Dental Association

- International Journal Oral and Maxillofacial Implants

Además, se realizó búsqueda de resúmenes pertinentes en la base de datos electrónica de las actas de la International Association for Dental Research (IADR) (desde su inicio hasta 2017), sin limitación de idiomas. Finalmente, se completó la búsqueda revisando manualmente las listas de referencias de los artículos incluidos para indagar la existencia de estudios adicionales no encontrados en la búsqueda electrónica.

\section{Criterios de selección para los estudios}

Se seleccionaron los ensayos clínicos aleatorizados controlados que midieron la ocurrencia de eventos hemorrágicos en pacientes bajo TACO sometidos a procedimientos de cirugía oral, tales como exodoncias, biopsias, cirugía periapical, exéresis de quistes y colocación de implantes, comparando con un grupo en el que se suspendió o modificó la terapia antitrombótica. 
Riesgo de hemorragia postquirúrgica en pacientes bajo tratamiento antitrombótico sometidos a cirugía oral: Revisión Sistemática y Metaanálisis.

Se excluyeron estudios descritos como ensayos clínicos no controlados, estudios observacionales, reportes de casos y cartas al editor.

\section{Desenlaces}

- Hemorragia posquirúrgica: sangrado del sitio quirúrgico prolongado y/o suficientemente considerable para que el paciente consulte espontáneamente al clínico tratante o a un servicio de urgencia.

- Evento tromboembólico: aparición de cuadro tromboembólico durante el seguimiento del paciente posterior a intervención quirúrgica.

\section{Mortalidad.}

\section{Selección de los estudios y Extracción de los datos}

Se realizó la selección de los estudios mediante dos etapas, en la primera etapa los artículos obtenidos de la búsqueda sistemática fueron enumerados y evaluados usando los criterios de selección preestablecidos, según el título y su resumen. Este proceso se llevó a cabo de forma independiente por dos revisores (AA y NY) que siguieron instrucciones especialmente diseñadas para esta etapa, siendo ampliamente inclusivo.

Se recuperaron todos los estudios seleccionados para continuar con la evaluación a texto completo por ambos revisores en forma independiente, seleccionando los que cumplieron con los criterios de inclusión.

Los desacuerdos se resolvieron por consenso y, si no se llegó a un consenso, un tercer revisor (JV) actuó como árbitro. Se incluye un diagrama de flujo para ilustrar los resultados de la actividad de búsqueda y el proceso de filtro y selección de los estudios para su inclusión en la revisión18. (Fig. 1)

Dos revisores, de forma independiente, extrajeron los datos de todos los estudios seleccionados, mediante un formulario creado en Microsoft Word Office para este fin, basado en las recomendaciones del Manual Cochrane para Revisiones Sistemáticas de Intervenciones (MCRSI) ${ }^{(19)}$.

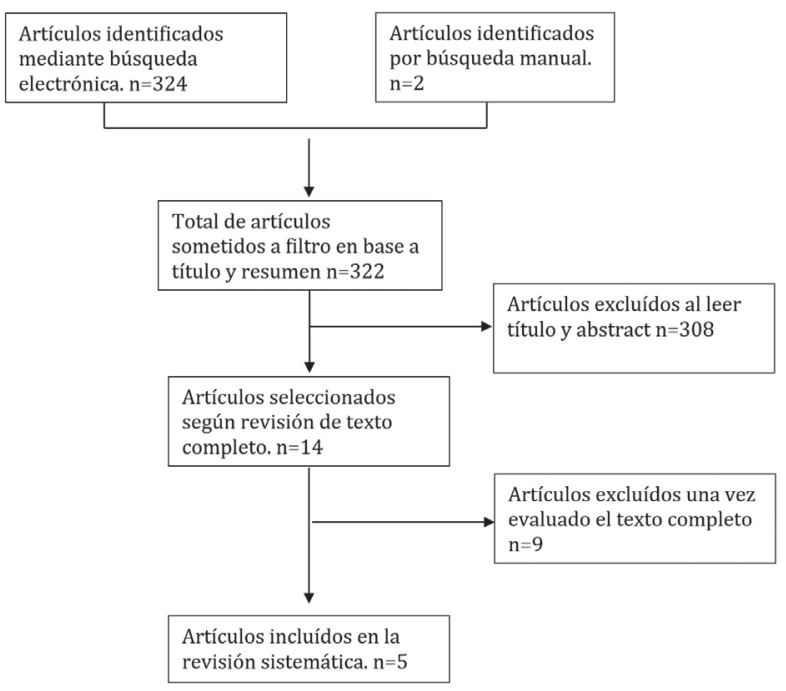

Figura 1. Diagrama de flujo: Identificación y selección de estudios.

El formulario utilizado incluyó principalmente la siguiente información:

- Características de los estudios: año y contexto clínico de realización, número de pacientes incluidos y razones de las pérdidas de seguimiento, número de grupos de tratamiento y control, intervención de interés, intervención control y cointervenciones.

- Características de los pacientes: edad, sexo, estado de salud, criterios de selección para la inclusión en el estudio.

- Información sobre los desenlaces: definiciones utilizadas por los autores y número total de pacientes por grupo y que presentaron el evento.

- Evaluación del riesgo de sesgo de los estudios incluidos.

Las discrepancias entre los datos fueron analizadas por los dos revisores y, de ser necesario, un tercer revisor actuó como árbitro.

El riesgo de sesgo de los estudios incluidos se evaluó mediante la Herramienta de riesgo de sesgo Cochrane ${ }^{(19)}$ para ensayos clínicos aleatorizados. Se utilizaron todos los ámbitos (dominios) de esta herramienta (generación de la secuencia, ocultamiento de la asignación, cegamiento de los participantes, personal y evaluadores de resultados, datos perdidos de los participantes, notificación selectiva de desenlaces y otras fuentes de sesgo) y se siguieron las instrucciones publicadas en el MCRSI para la evaluación de cada dominio y para la realización de la evaluación del riesgo de sesgo general.

Esta evaluación fue realizada por dos revisores de forma independiente, basándose en el texto completo de los estudios incluidos. Se discutieron los desacuerdos entre los revisores y se debió alcanzar un consenso para clasificar un estudio como bajo, alto o poco claro riesgo de sesgo.

\section{Medición y análisis de los desenlaces}

- Desenlace hemorragia posquirúrgica: se consideró como una variable dicotómica y se calculó el cociente de riesgo o riesgo relativo $(R R)$ y su correspondiente intervalo de confianza (IC) del 95\%.

- Desenlace evento tromboembólico y mortalidad: se planificó analizar los datos de manera dicotómica con el cálculo del RR e IC de 95\%.

\section{Evaluación de la heterogeneidad de los estudios}

Se utilizó la prueba de $\mathrm{Chi}^{(2)}$ para determinar la presencia de heterogeneidad estadística, utilizando un nivel de significancia de 0,1 . La cuantificación de la heterogeneidad entre los estudios se hizo mediante el uso del estimador $I^{(2)}$, y su interpretación se basó en las recomendaciones Cochrane ${ }^{(19)}$ : es decir, un ${ }^{(2)}$ entre el $0 \%$ y el $40 \%$ se consideró como heterogeneidad poco importante entre los ensayos; $30 \%$ a $60 \%$ podría representar heterogeneidad moderada; $50 \%$ a $90 \%$ podría representar heterogeneidad significativa; y $75 \%$ a $100 \%$ podría representar una heterogeneidad considerable.

\section{Síntesis de los datos}

Se realizó un resumen narrativo de las características de los estudios incluidos, además de un metaanálisis de los datos extraídos de los estudios utilizando el Programa RevMan 5.320, utilizando un modelo de efectos aleatorios.

\section{Evaluación de la calidad de la evidencia}

Después de realizar todo este proceso, se calificó la calidad de la evidencia (Confianza en las estimaciones) para el resultado (sangramiento) usando el enfoque de GRADE. Se utilizaron los cinco criterios de este enfoque: diseño de los estudios que contribuyen al resultado, riesgo de sesgo, inconsistencia, imprecisión e evidencia indirecta ${ }^{(21)}$. Se incluye un perfil de evidencia GRADE en los resultados

\section{RESULTADOS}

\section{Resultados de la búsqueda}

La búsqueda resultó en 322 referencias las que se filtraron según el título y el resumen (first screening) según los criterios de inclusión y exclusión por parte de dos revisores (AA, NY). Los detalles de los resultados de cada etapa de búsqueda y screening se pueden encontrar en la Figura 1.

Resultado de lo anterior se seleccionaron 14 artículos, lo cuales fueron revisados a texto completo y en un segundo tiempo, seleccionándose un total de 5 estudios, los cuales se incluyeron en esta revisión ${ }^{(22-26)}(\mathrm{Kappa}=$ 0,77 ), como se muestran en la Tabla 2.

\section{Estudios excluidos}

Un total de 9 estudios de los que se sometieron a evaluación de texto completo fueron excluidos de esta revisión. Dos de ellos eran duplicados y correspondían al mismo estudio, pero con un tamaño de muestra menor realizado el año anterior ${ }^{(27,28)}$, mientras que otros dos correspondían a cartas al editor ${ }^{(29,30)}$. Por último, se excluyeron dos estudios porque no contaron con las comparaciones de interés ${ }^{(31,32)}$ y un estudio en donde no se suspendió el TACO en ninguno de los grupos y sólo se redujo la dosis del fármaco ${ }^{(33)}$.

\section{Descripción de los estudios incluidos}

De los 5 estudios incluidos, tres se llevaron a cabo en Europa ${ }^{(24-26)}$, uno en Norteamérica ${ }^{(23)}$ y otro en Asia ${ }^{(22)}$. Uno de los estudios se realizó en una Facultad de Odontología23, dos se realizaron en un Hospital Clínico ${ }^{(24,25)}$, otro se llevó a cabo en un Hospital docente ${ }^{(26)}$ y el estudio restante no especifica claramente el lugar de realización ${ }^{(22)}$.

\section{Participantes}

Los estudios reclutaron entre $35^{(23)}$ y $214^{(22)}$ participantes. Tres de los estudios contaron con una proporción mayor de hombres que de mujeres y con una edad media mayor a 60 años, mientras que en un estudio la proporción de mujeres fue mayor y la edad media de los pacientes fue de 51 años ${ }^{(22)}$ En el estudio restante no se especificó ni edad ni sexo ${ }^{(23)}$.

El tipo y número de intervenciones de cirugía oral menor variaron de acuerdo a lo reportado por cada estudio. Se realizaron sólo exodoncias en dos estudios ${ }^{(22,25)}$ y en los tres restantes se realizaron otros procedimientos de cirugía oral menor además de exodoncias, como alveoloplastías, exéresis quísticas, colocación de implantes endoóseos, y biopsias de tejido blando.

Los 5 estudios informaron criterios de inclusión de los pacientes para el estudio. Cuatro de los ensayos incluidos informaron criterios de exclusión, teniendo en común entre ellos la decisión de excluir del estudio pacientes con enfermedad hepática. El estudio restante no entregó información sobre criterios de exclusión de pacientes ${ }^{(23)}$.

\section{Intervenciones}

Tres de los ensayos contaron con dos grupos de estudio paralelo ${ }^{(24-26)}$, 
Tabla 2. Características de los estudios incluidos. $\mathrm{N}$ pacientes $=$ Número de pacientes; Sgto =Seguimiento; $w=W a r f a r i n a ; s=S u t u r a ; ~ E H=E n f e r m e d a d$ Hepática; T=Grupo tratamiento; C=Grupo control; TXA=Ácido tranexámico; (+) =Continuación; (-) =Detención.

\begin{tabular}{|c|c|c|c|c|c|c|c|c|}
\hline Estudios & $\begin{array}{l}\text { Criterios de } \\
\text { inclusión de } \\
\text { Pacientes }\end{array}$ & $\begin{array}{l}\text { Criterios de } \\
\text { exclusión de } \\
\text { Pacientes }\end{array}$ & $\stackrel{\text { N }}{\text { Pacientes }}$ & $\begin{array}{l}\text { Intervenciones } \\
\text { (Por cada } \\
\text { grupo) }\end{array}$ & $\begin{array}{c}\text { INR } \\
\text { Promedio }\end{array}$ & $\begin{array}{c}\text { Procedimientos } \\
\text { de cirugía oral } \\
\text { menor }\end{array}$ & Cointervenciones & $\begin{array}{l}\text { Sgto. } \\
\text { (Días) }\end{array}$ \\
\hline \multirow{4}{*}{$\begin{array}{c}\text { Al } \\
\text { Mubarak } \\
2007\end{array}$} & \multirow{4}{*}{$\begin{array}{l}\text { TACO con W, } \\
\text { dosis de } 2-10 \\
\text { mg/día durante } \\
\text { más de } 1 \text { año } \\
\text { e indicación de } \\
\text { exodoncia. }\end{array}$} & \multirow{4}{*}{$\begin{array}{c}\text { EH o renal } \\
\text { crónica, } \\
\text { consumo de } \\
\text { fármacos que } \\
\text { afectan función } \\
\text { hepática o } \\
\text { hemostasia }\end{array}$} & \multirow{4}{*}{214} & $\begin{array}{c}\text { (1) -W-S (48 } \\
\text { pacientes) }\end{array}$ & $1.8 \pm 0.4$ & \multirow{4}{*}{ Exodoncias } & \multirow{4}{*}{ No reportado } & \multirow{4}{*}{1,3 y 7} \\
\hline & & & & $\begin{array}{c}(2)+w-s(58 \\
\text { pacientes) }\end{array}$ & $2.4 \pm 0.5$ & & & \\
\hline & & & & $\begin{array}{c}\text { (3) }-w+s(56 \\
\text { pacientes) }\end{array}$ & $1.9 \pm 0.4$ & & & \\
\hline & & & & $\begin{array}{c}(4)+w+s(52 \\
\text { pacientes) }\end{array}$ & $2.7 \pm 0.4$ & & & \\
\hline \multirow{3}{*}{$\begin{array}{l}\text { Campbell } \\
2000\end{array}$} & \multirow{3}{*}{$\begin{array}{c}\text { TACO e } \\
\text { indicación de } \\
\text { cirugía oral } \\
\text { menor. }\end{array}$} & \multirow{3}{*}{ No reportado } & \multirow{3}{*}{35} & $\begin{array}{c}\text { T: +TACO (12 } \\
\text { pacientes) }\end{array}$ & $2(1.2-2.9)$ & \multirow{3}{*}{$\begin{array}{l}\text { Exodoncias, } \\
\text { alveoloplastías y } \\
\text { cirugía de tejido } \\
\text { blando (biopsia, } \\
\text { frenectomía). }\end{array}$} & \multirow{3}{*}{ No reportado } & \multirow{3}{*}{1} \\
\hline & & & & $\begin{array}{l}\text { C: }- \text { TACO } 72 \text { a } \\
96 \text { horas antes } \\
\text { (13 pacientes) }\end{array}$ & $2(1.1-3)$ & & & \\
\hline & & & & $\begin{array}{l}\text { Base: Nunca } \\
\text { TACO (10 } \\
\text { pacientes) }\end{array}$ & - & & & \\
\hline \multirow{2}{*}{$\begin{array}{c}\text { Cannon } \\
2003\end{array}$} & \multirow{2}{*}{$\begin{array}{l}\text { TACO con W } \\
\text { derivados para } \\
\text { cirugía oral. }\end{array}$} & \multirow{2}{*}{$\begin{array}{l}\text { INR fuera } \\
\text { de rango } \\
\text { terapéutico 2-4, } \\
\text { historia de EH } \\
\text { o consumo de } \\
\text { fármacos que } \\
\text { afectan función } \\
\text { hepática. }\end{array}$} & \multirow[b]{2}{*}{70} & $\begin{array}{l}\text { T: +TACO (35 } \\
\text { pacientes) }\end{array}$ & $3.4(2.1-4)$ & \multirow{2}{*}{$\begin{array}{l}\text { Exodoncias, } \\
\text { exéresis } \\
\text { quísticas, } \\
\text { biopsias, cierre } \\
\text { de fístulas } \\
\text { oroantrales. }\end{array}$} & \multirow{2}{*}{ 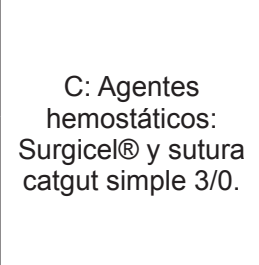 } & \multirow[b]{2}{*}{3 y 5} \\
\hline & & & & $\begin{array}{l}\text { C: -TACO } 2 \\
\text { días antes (35 } \\
\text { pacientes) }\end{array}$ & & & & \\
\hline \multirow[b]{2}{*}{$\begin{array}{l}\text { Evans } \\
2002\end{array}$} & \multirow{2}{*}{$\begin{array}{l}\text { TACO con W e } \\
\text { indicación de } \\
\text { exodoncias }\end{array}$} & \multirow{2}{*}{$\begin{array}{c}\text { Sin } \\
\text { consentimiento } \\
\text { informado, sin } \\
\text { acceso a teléfono, } \\
\text { INR }>4 \text {, EH o } \\
\text { coagulopatías, } \\
\text { imposibilidad } \\
\text { de asistir a } \\
\text { seguimiento. }\end{array}$} & \multirow[b]{2}{*}{109} & $\begin{array}{c}\text { T: + TACO (57 } \\
\text { pacientes) }\end{array}$ & $\begin{array}{c}2.5(1.2- \\
4.7)\end{array}$ & \multirow[b]{2}{*}{ Exodoncias } & \multirow{2}{*}{$\begin{array}{c}\text { Compresión } \\
\text { con esponjas } \\
\text { de oxicelulosa } \\
\text { (Surgicel囚) y } \\
\text { sutura poliglactina } \\
9103 / 0 \text { (Vicryl®). }\end{array}$} & \multirow[b]{2}{*}{7} \\
\hline & & & & $\begin{array}{c}\text { C: - TACO } 2 \\
\text { días antes (52 } \\
\text { pacientes) }\end{array}$ & $\begin{array}{c}1.6(1.2- \\
2.3)\end{array}$ & & & \\
\hline \multirow[b]{2}{*}{$\begin{array}{l}\text { Sacco } \\
2007\end{array}$} & \multirow{2}{*}{$\begin{array}{l}\text { Cualquier edad } \\
\text { y género, en } \\
\text { TACO durante } \\
\text { al menos } 2 \\
\text { semanas por } \\
\text { cualquier razón } \\
\text { médica, INR 2-4. }\end{array}$} & \multirow{2}{*}{$\begin{array}{l}\text { Bajo recuento } \\
\text { plaquetario } \\
\text { (menos de } \\
\text { 100.000), EH y } \\
\text { renal, limitada } \\
\text { capacidad física } \\
\text { y psicológica } \\
\text { que impidiera } \\
\text { continuar hasta } \\
\text { finalización del } \\
\text { estudio. }\end{array}$} & \multirow[b]{2}{*}{131} & $\begin{array}{l}\mathrm{T}: \text { +TACO (65 } \\
\text { pacientes) }\end{array}$ & $2.8 \pm 0.50$ & \multirow[b]{2}{*}{$\begin{array}{l}\text { Exodoncias, } \\
\text { colocación } \\
\text { de implantes, } \\
\text { exéresis } \\
\text { quísticas. }\end{array}$} & \multirow{2}{*}{$\begin{array}{c}\text { T: Medidas } \\
\text { hemostáticas: } \\
\text { gelita y esponjas } \\
\text { de oxicelulosa, } \\
\text { aplicación local } \\
\text { de TXA en lecho } \\
\text { quirúrgico y luego } \\
\text { como enjuague } \\
\text { bucal, cada } 6 \\
\text { horas durante } 2 \\
\text { días. }\end{array}$} & \multirow[b]{2}{*}{7} \\
\hline & & & & $\begin{array}{l}\text { C: Reducción } \\
\text { dosis TACO } \\
\text { con INR } 1.8 \text { (66 } \\
\text { pacientes) }\end{array}$ & $2.74 \pm 0.72$ & & & \\
\hline
\end{tabular}

mientras que un estudio tuvo tres grupos ${ }^{(23)}$ y otro tuvo cuatro grupos ${ }^{(22)}$.

Dos de los ensayos que contaron con dos grupos en estudio, evaluaron los efectos de la mantención de la terapia con warfarina frente a los efectos de la interrupción de ésta dos días antes de la cirugía ${ }^{(24,25)}$, mientras que el tercero evaluó los efectos de la mantención del TACO versus los efectos de la reducción de la dosis de anticoagulante manteniendo un INR entre 1,5 y $2^{(26)}$. El ensayo que contaba con tres grupos midió los efectos de la mantención del TACO en régimen regular, comparado con otro grupo que detuvo su terapia 72 a 96 horas antes y, adicionalmente, incluyó un grupo de pacientes que nunca había consumido anticoagulantes para evaluar el sangrado basal(23). Por último, el ensayo que contaba con cuatro grupos de estudio analizó los efectos de la mantención del TACO en pacientes que recibieron sutura y en pacientes que no la recibieron, en comparación con dos grupos de pacientes que detuvieron su régimen anticoagulante dos días antes de la cirugía, habiendo un grupo recibido sutura y el otro no.

Dos de los estudios informaron la realización de cointervenciones en solo uno de los grupos ${ }^{(24,26)}$

\section{Desenlaces}

Todos los estudios midieron hemorragia posquirúrgica: si bien contaron con diferencias en los métodos de cuantificación, todos tuvieron en común la evaluación por parte del profesional, monitorizando al paciente las primeras 24 horas de realizada la cirugía.

Cuatro estudios midieron la ocurrencia de cuadros hemorrágicos durante un determinado tiempo de espera del paciente en el recinto clínico posterior a la cirugía: durante 2 horas de espera en el lugar ${ }^{(26)}, 1$ hora de espera en el lugar ${ }^{(22)}, 30$ minutos después del procedimiento ${ }^{(24)}, 20$ minutos antes de dar el alta ${ }^{(25)}$.

Ninguno de los estudios reportó la ocurrencia de eventos tromboembólicos ni de mortalidad posterior a la intervención.

\section{Riesgo de sesgo en los estudios incluidos}

La evaluación del riesgo de sesgo se ilustra en las figuras 2 y 3.

En general, ninguno de los estudios cumplió cabalmente con bajo riesgo de sesgo en todos los ámbitos abarcados en el análisis crítico. Si bien todos cumplieron con un bajo riesgo en el aspecto relacionado a la notificación 


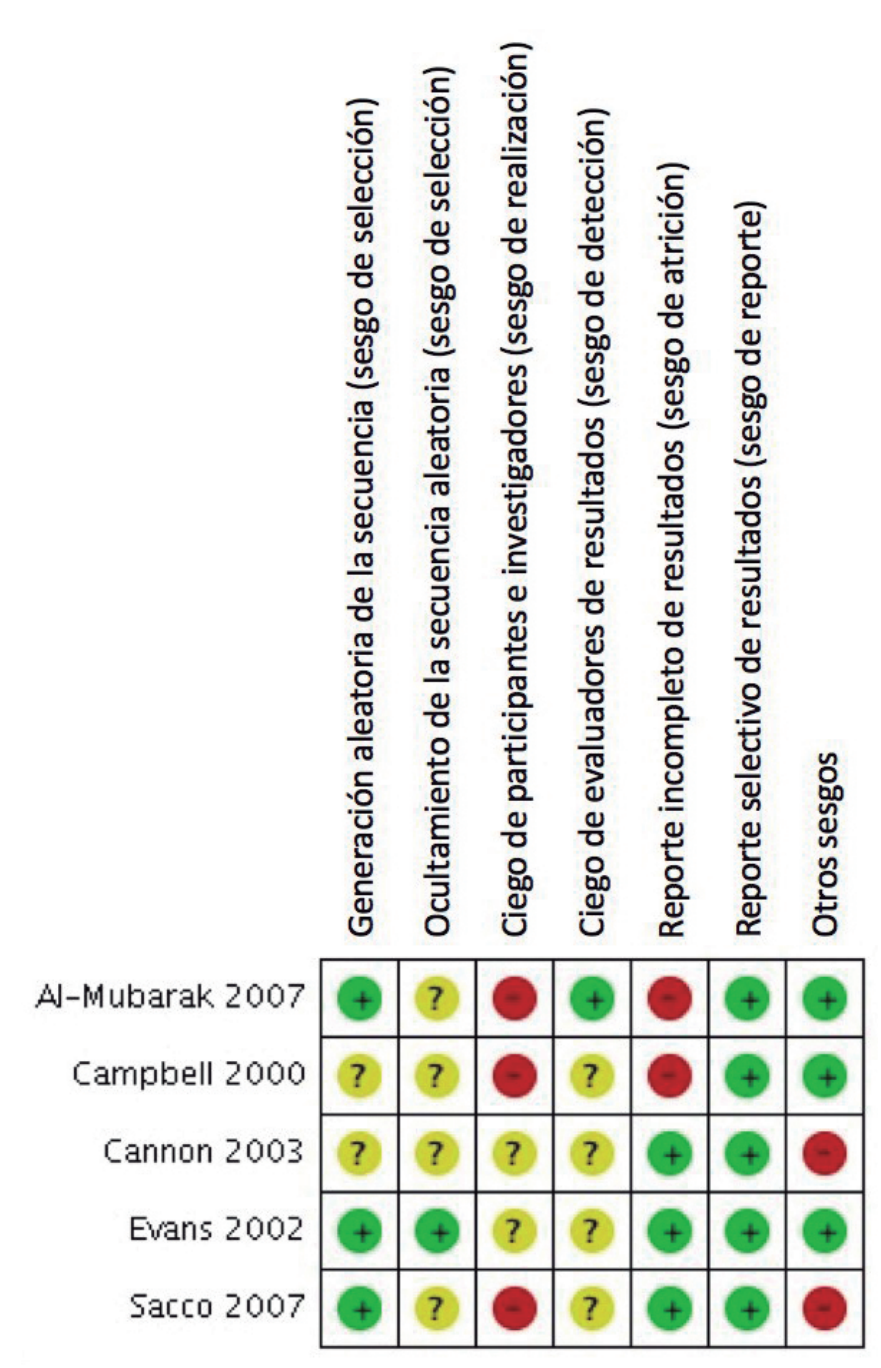

Figura 2. Figura 2 Resumen de riesgo de sesgo: Juicio de los revisores para los dominios evaluados en cada estudio incluido.

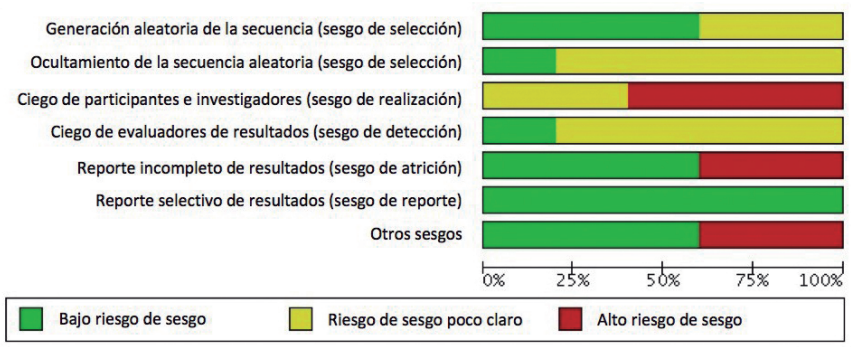

Figura 3. Gráfico de riesgo de sesgo: juicio de los revisores en cada dominio del riesgo de sesgo de todos los estudios incluídos, expresado como porcentajes.

selectiva de resultados, en otros como el cegamiento, tanto de pacientes, como de participantes del estudio, todos los estudios presentaron riesgo poco claro o alto.

Otras posibles fuentes de sesgo aparte de las analizadas en forma directa fueron detectadas sólo en dos estudios en relación a cointervenciones aplicadas sólo en uno de los grupos en estudio, principalmente referido a medidas hemostáticas locales como el uso de esponjas de celulosa, sutura y ácido tranexámico, pudiendo de esta forma modificar la ocurrencia de la hemorragia ${ }^{(24,26)}$.

\section{Desenlace de hemorragia post quirúrgica}

Este desenlace fue informado por 5 estudios que incluyeron un total de 549 pacientes y 84 eventos hemorrágicos en total. Mediante la realización del metanálisis se pudo observar que la mantención de la terapia antitrombótica aumenta el riesgo de sangrado postquirúrgico estimado en un RR 1.41 [0.93 - 2.16] $p=0,11$, con un intervalo de confianza del 95\%, comparado con la suspensión de la terapia antitrombótica. Sin embargo, esta diferencia no es estadísticamente significativa. Respecto a la heterogeneidad, la prueba Chi cuadrado arrojó un valor $p$ de 0,39 asociado a un estimador $I^{(2)}$ de $3 \%$.

La calidad de la evidencia para este desenlace es baja, dado el elevado riesgo de sesgo de los estudios y la falta de precisión de sus resultados.

\section{Desenlaces de evento tromboembólico y mortalidad}

Ninguno de los estudios analizados informó la ocurrencia de alguno de estos desenlaces dentro de los grupos estudiados.

Se calificó el efecto protector de suspender la terapia anticoagulante sobre el sangrado perioperatorio en pacientes sometidos a procedimientos de cirugía oral menor como evidencia de baja calidad según los criterios GRADE $^{(21)}$ (Tabla 3).

\section{DISCUSIÓN}

El resultado final arrojado por el metaanálisis (figura 4) indica que la mantención de la terapia antitrombótica aumenta el riesgo de sangrado postquirúrgico estimado en un $41 \%(R R=1.41$ [0.93 - 2.16]) con un intervalo de confianza del $95 \%$, comparado con la suspensión de la terapia antitrombótica, sin embargo, dicho intervalo se encuentra en la zona de no efecto, resultando en un indicador que no es estadísticamente significativo. En este mismo sentido, cuatro de los cinco ECR incluidos en este metaanáisis obtuvieron resultados que, desde el punto de vista de sus indicadores puntuales favorecen la suspensión de la terapia antitrombótica y lo hacen con una baja heterogeneidad estadística $\left({ }^{(2)}=3 \% \mathrm{p}=0,39\right)$, pero igualmente el intervalo de confianza pasa por el no efecto.

Por otro lado, los estudios incluidos no contaron con una descripción adecuada del cegamiento de los pacientes y del personal, ni tampoco mencionan lo métodos por los cuales se llevó a cabo el cegamiento de los evaluadores de eventos, lo cual podría estar asociado a una estimación más exagerada del efecto de la intervención ${ }^{(19)}$, dado que el conocimiento de la intervención realizada por cualquiera de los participantes del estudio podría modificar tanto la respuesta del paciente al tratamiento, como la valoración que los profesionales pudieran hacer respecto del desenlace analizado, en este caso, la ocurrencia de hemorragia postquirúrgica.

En una revisión sistemática realizada previamente por Nematullah el $2009^{(1)}$ se obtuvo un resultado similar, cuyo estimador puntual favorecía la mantención del TACO, pero igualmente con un intervalo de confianza que incluye la zona del no efecto en su recorrido, sin embargo, dicho artículo incluyó dos estudios ${ }^{(27,34)}$ correspondientes a ensayos clínicos que no se limitaban a la intervención examinada en este caso, sino que agregaban cointervenciones con agentes como la heparina y el ácido tranexámico en alguno de sus grupos, además de dejar fuera estudios relevantes que si llevaban a cabo la comparación de intervenciones consideradas en este trabajo $0^{(16,17,19)}$

La presente revisión sistemática incluyó una búsqueda amplia, sin restricciones de lenguaje, así como un método estandarizado para el screening de los títulos y resúmenes y una selección de estudios realizada en forma independiente y duplicada. La evaluación del riesgo de sesgo se realizó mediante criterios establecidos y por evaluadores independientes, según la metodología propuesta en el manual Cochrane revisiones sistemáticas de intervenciones. Sin embargo, no se incluyeron resultados de estudios no publicados, literatura gris, dada la falta de respuesta de los autores de los artículos seleccionados ante la solicitud de envío de datos no incluidos en sus artículos $u$ otros en desarrollo. Es relevante mencionar en este sentido que a pesar de haberse realizado una búsqueda sin limitación de idiomas y a pesar de la variedad de nacionalidades de los autores de los artículos incluidos en esta revisión, sólo fue posible incluir ensayos publicados en inglés, lo cual pone de manifiesto la preferencia que existe por parte de los autores de estudios con alto nivel de evidencia por publicar en este y no otro idioma, posiblemente por el bajo impacto atribuido a las revistas que difunden en otras lenguas.

A la luz del metaanálisis realizado en esta revisión sistemática, podemos mencionar objetivamente que existe una tendencia hacia un aumento de los eventos hemorrágicos en los grupos de pacientes en los cuales no se lleva a cabo una modificación de la terapia anticoagulante oral frente a procedimientos de cirugía oral. Sin embargo, el aumento no es estadísticamente significativo y según lo reportado por los diferentes estudios, en ningún caso se reportaron sangrados incoercibles ni estuvo comprometida la integridad de los pacientes a causa de otras reacciones adversas, siendo las medidas locales de hemostasia un recurso suficiente para la contención de los episodios hemorrágicos ${ }^{(10,11)}$, resultando finalmente en una diferencia que clínicamente tampoco es significativa.

Si bien es importante tener en cuenta el mayor riesgo de hemorragia que conllevan estos pacientes ${ }^{(33,34)}$, los resultados de esta revisión ponen a la práctica clínica de la cirugía oral en un escenario en el cual debe cuidarse la prolijidad de la técnica quirúrgica y la aplicación de las estrategias locales de control del sangrado, tales como el uso de esponjas hemostáticas, su- 
Tabla 3. Calidad de la evidencia GRADE:

\section{Suspender TACO comparado con Mantener TACO para Pacientes sometidos a cirugía oral}

Paciente o población : Pacientes sometidos a cirugía oral

Configuración: Revisión sistemática

Intervención : Suspender TACO

Comparación: Mantener TACO

\begin{tabular}{|c|c|c|c|c|c|c|}
\hline \multirow{2}{*}{$\begin{array}{l}\text { Resultado } \\
\text { № de participantes } \\
\text { (Estudios ) }\end{array}$} & \multirow{2}{*}{$\begin{array}{l}\text { Efecto } \\
\text { relativo } \\
(95 \% \mathrm{Cl})\end{array}$} & \multicolumn{3}{|c|}{ Efectos absolutos anticipados $(95 \% \mathrm{Cl})$} & \multirow[b]{2}{*}{ Certeza } & \multirow[b]{2}{*}{ Qué pasa } \\
\hline & & $\begin{array}{l}\text { Sin Suspender } \\
\text { TACO }\end{array}$ & $\begin{array}{l}\text { Con Suspender } \\
\text { TACO }\end{array}$ & Diferencia & & \\
\hline $\begin{array}{l}\text { Sangrado postoperatorio } \\
\text { № de participantes : } 549 \\
\text { ( } 5 \text { Experimentos controlados } \\
\text { aleatorios [ECAs]) }\end{array}$ & $\begin{array}{l}\text { RR } 1.41 \\
(0.93 \mathrm{a} \\
2.16)\end{array}$ & $12.6 \%$ & $\begin{array}{c}17.8 \% \\
(11.7 \text { a } 27.2)\end{array}$ & $\begin{array}{l}5.2 \% \text { más } \\
(0,9 \text { menos a } \\
14,6 \text { más })\end{array}$ & $\begin{array}{l}\oplus \oplus \bigcirc \bigcirc \\
\text { BAJA } a, b\end{array}$ & \\
\hline $\begin{array}{l}\text { Eventos tromboembólicos - no } \\
\text { reportado }\end{array}$ & - & - & - & - & - & \\
\hline Mortalidad - no reportado & - & - & - & - & - & \\
\hline
\end{tabular}

El riesgo en el grupo de intervención (y su intervalo de confianza del 95\%) se basa en el riesgo asumido en el grupo de comparación y en el efecto relativo de la intervención (y su intervalo de confianza del 95\%).

Cl: Intervalo de confianza ; RR: Razón de riesgo

\section{GRADE Working Group grades of evidence}

Certeza Alta: Estamos muy confiados de que el verdadero efecto esta cercano a la estimacion del efecto.

Certeza Moderada: Estamos moderadamente confiados de la estimacion del efecto:El verdadero efecto probablemente es cercano al estimador del efecto, pero existe una posibilidad de que sea sustancialmente diferente.

Baja certeza: Nuestra confianza en la estimación del efecto es limitada: El verdadero efecto podría ser sustancialmente diferente al estimador del efecto.

Muy baja certeza: Tenemos muy poca confianza en la estimación del efecto: Es probable que el verdadero efecto sea sustancialmente diferente del efecto estimado.

\section{Explicaciones}

a. Serios problemas en evaluación de riesgo de sesgo porque los estudios presentaron un riesgo alto o poco claro en el dominio de cegamiento de personal, se detectó riesgo alto en dos estudios en reporte incompleto de resultados y se detectaron otras fuentes de riesgo de sesgo alto en otros dos estudios.

b. Serios problemas de imprecisión: intervalo de confianza de 95\% amplio, sugiriendo grandes beneficios o perjuicios. Poca cantidad de eventos ( $n=84$ )

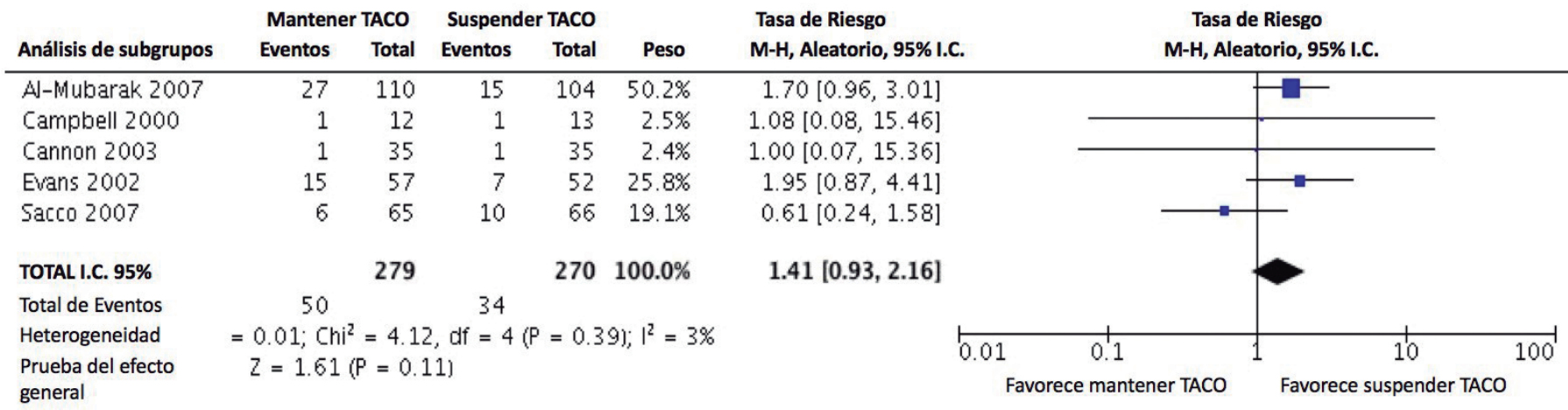

Figura 4. Forest plot

tura y la compresión local. Es importante también el llevar a cabo controles inmediatos post-quirúrgicos, con el fin de pesquisar precozmente posibles complicaciones en pacientes en los que no se ha modificado la terapia anticoagulante, ya que la mayoría de los episodios reportados en estos estudios fueron objetivados por el equipo quirúrgico en un control previo al alta del paciente desde los centros donde las intervenciones fueron realizadas.

Debemos destacar la importancia de realizar el control previo del tiempo de protrombina e INR de los pacientes. Todos los estudios incluidos en esta revisión fueron examinados para comprobar que los pacientes se encontraran en el rango terapéutico (INR 2 a 3), lo cual debería ser parte de la práctica habitual al enfrentarse a este tipo de escenario clínico $22,35,36)$.

En el marco de las opciones farmacológicas actuales para la anticoagulación, es importante poner de manifiesto que todos los estudios que engloba esta revisión sistemática se refieren a anticoagulantes antagonistas de la vitamina $\mathrm{K}$ derivados de la warfarina, por lo que no es posible extrapolar sus resultados a aquellos pacientes que utilizan nuevos anticoagulantes, tales como el Dabigatrán, Rivaroxabán, Apixabán, etc.

Si bien es cierto que la mayoría de los estudios que cumplieron con los criterios de inclusión para esta revisión sistemática, no cumplen con la mayoría de los criterios considerados para la evaluación de su validez interna, 
aun así, nos entregan una idea general de la tendencia que existe en la clínica respecto de la posibilidad de ocurrencia de hemorragias post-quirúrgicas en este tipo de pacientes. Sin embargo, a la vez se pone de manifiesto la completa ausencia de reporte en estos estudios sobre desenlaces como eventos tromboembólicos o mortalidad por cualquier causa asociada a las intervenciones comparadas en los ensayos clínicos, lo cual no justificaría la realización de nuevos ensayos clínicos al respecto.

A pesar de lo anterior, se debería mantener un seguimiento adecuado de este tema, por lo que es importante el desarrollo de estudios observacionales en el tiempo, con el fin de confirmar la tendencia demostrada en esta revisión, o bien, alertar sobre comportamientos del desenlace que difieran de ella, los cuales sin duda deberían transformarse en nuevos motivos de investigación para explicar las diferencias en la dirección de los resultados.

Por otra parte, es importante el desarrollo de estudios respecto a los nuevos anticoagulantes orales, de los cuales no existe ningún ensayo clínico que los incluya y oriente objetivamente y con un alto nivel de evidencia a un comportamiento terapéutico específico en caso de pacientes que los utilicen y que necesiten ser sometidos a intervenciones de cirugía oral.
Finalmente, la presente revisión sistemática muestra una tendencia a una mayor cantidad de episodios de hemorragia post cirugía oral en pacientes que continúan su terapia anticoagulante oral con antagonistas de vitamina $\mathrm{K}$, comparado con pacientes que la interrumpen o modifican, encontrándose siempre dentro del rango terapéutico de INR entre 2 y 3 . Sin embargo, dicha diferencia no es estadística ni clínicamente significativa. Además, no se reportan eventos que pongan en riesgo la integridad o la vida de los pacientes y los episodios hemorrágicos ocurridos fueron controlados exitosamente con medidas hemostáticas locales.

\section{CONFLICTO DE INTERÉS}

Los autores declaran no tener conflictos de interes, ni fuentes de financiamiento

Proyecto registrado en la Dirección de Investigación de la Facultad de Odontología, Universidad de Chile. PRI-ODO 15-007.

\section{Bibliografía}

1. Nematullah A, Alabousi A, Blanas N, Douketis JD, Sutherland SE. Dental surgery for patients on anticoagulant therapy with warfarin: a systematic review and metaanalysis. J Can Dent Assoc. 2009;75(1):41-41.

2. Wadelius M, Pirmohamed M. Pharmacogenetics of warfarin: current status and future challenges. Pharmacogenomics J. 2007;7(2):99-111.

3. Lip GYH, Lane DA. Stroke prevention in atrial fibrillation: a systematic review. JAMA. 2015;313(19):1950-62

4. Manolopoulos VG, Ragia G, Tavridou A. Pharmacogenetics of coumarinic oral anticoagulants. Pharmacogenomics. 2010;11(4):493-6

5. Berkovits A, Aizman A, Zúñiga P, Pereira J, Mezzano D. Nuevos anticoagulantes orales. Rev Méd Chile. 2011;139(10):1347-55

6. Doonquah L, Mitchell AD. Oral surgery for patients on anticoagulant therapy: current thoughts on patient management. Dent Clin North Am. 2012;56(1):25-41

7. Prandoni P, Noventa F, Ghirarduzzi A, Pengo V, Bernardi E, Pesavento R, et al The risk of recurrent venous thromboembolism after discontinuing anticoagulation in patients with acute proximal deep vein thrombosis or pulmonary embolism. A prospective cohort study in 1,626 patients. Haematologica. 2007:92(2):199-205.

8. Toscano LCNJ, Mumford CJ, Turner CB. Dental management of the anticoagulated patient. Clinical update. 2006; 28:9-10.

9 Pedemonte T C, Montini C, Castellón L. Manejo de pacientes en tratamiento con anticoagulantes orales previo a cirugía oral. Rev Odontológica Mex [Internet] 2005;9(4)171-177. [cited 2016 Jan 11]; Available from: http://revistas.unam.mx/ index.php/rom/article/view/15952

10. Soares ECS, Costa FWG, Bezerra TP, Nogueira CBP, de Barros Silva PG, Batista SHB, et al. Postoperative hemostatic efficacy of gauze soaked in tranexamic acid, fibrin sponge, and dry gauze compression following dental extractions in anticoagulated patients with cardiovascular disease: a prospective, randomized study. Oral Maxillofac Surg. 2015;19(2):209-16.

11. Weltman NJ, Al-Attar Y, Cheung J, Duncan DP, Katchky A, Azarpazhooh A, et al. Management of dental extractions in patients taking warfarin as anticoagulant treatment: a systematic review. J Can Dent Assoc. 2015;81:f20

12. Büller HR, Agnelli G, Hull RD, Hyers TM, Prins MH, Raskob GE. Antithrombotic therapy for venous thromboembolic disease: the Seventh ACCP Conference on Antithrombotic and Thrombolytic Therapy. Chest. 2004;126(3 Suppl):401s-428s

13. Salem DN, Stein PD, Al-Ahmad A, Bussey HI, Horstkotte D, Miller N, et al Antithrombotic therapy in valvular heart disease--native and prosthetic: the Seventh ACCP Conference on Antithrombotic and Thrombolytic Therapy. Chest. 2004;126(3 Suppl):457s-482s.

14. Nishimura RA, Otto CM, Bonow RO, Carabello BA, Erwin JP, Guyton RA, et al. AHA/ACC guideline for the management of patients with valvular heart disease: a report of the American College of Cardiology/American Heart Association Task Force on Practice Guidelines. J Thorac Cardiovasc Surg. 2014;148(1):e1-132

15. Murphy J, Twohig E, McWilliams SR. Dentists' approach to patients on antiplatelet agents and warfarin: a survey of practice. J Ir Dent Assoc. 2010;56(1):28-31 16. Dewan K, Vithlani V, Patel N, Warren K. A study to assess management of patients on warfarin by general dental practitioners (GDPS) in the west midlands. Dental Update. 2012;39(8):578-587.

17. Moher D, Liberati A, Tetzlaff J, Altman DG, The PRISMA Group. Preferred Reporting Items for Systematic Reviews and Meta-Analyses: The PRISMA Statement. PLoS Med. 2009;6(7):e1000097.

18. Liberati A, Altman DG, Tetzlaff J, Mulrow C, Gøtzsche PC, loannidis JPA, et al. The PRISMA statement for reporting systematic reviews and meta-analyses of studies that evaluate health care interventions: explanation and elaboration. J Clin Epidemiol. 2009;62(10):e1-34.
19. Higgins JP, Green S. Cochrane handbook for systematic reviews of interventions. Vol. 5. Wiley Online Library; 2008.

20. Review Manager (RevMan) [Computer program]. Version 5.3. Copenhagen: The Nordic Cochrane Centre, The Cochrane Collaboration, 2014.

21. Guyatt GH OA, Vist GE, Kunz R, Falck-Ytter Y, Alonso-Coello P, Schünemann $\mathrm{HJ}$; GRADE Working Group. GRADE: an emerging consensus on rating quality of evidence and strength of recommendations. BMJ. 2008;336(7650):924-6.

22. Al-Mubarak S, Al-Ali N, Abou-Rass M, Al-Sohail A, Robert A, Al-Zoman K, et al. Evaluation of dental extractions, suturing and INR on postoperative bleeding of patients maintained on oral anticoagulant therapy. Br Dent J. 2007;203(7):e15; discussion 410-1.

23. Campbell JH, Alvarado F, Murray RA. Anticoagulation and minor oral surgery: should the anticoagulation regimen be altered? J Oral Maxillofac Surg. 2000:58(2):131-5; discussion 135-6

24. Cannon PD, Dharmar VT. Minor oral surgical procedures in patients on oral anticoagulants--a controlled study. Aust Dent J. 2003;48(2):115-8.

25. Evans IL, Sayers MS, Gibbons AJ, Price G, Snooks H, Sugar AW. Can warfarin be continued during dental extraction? Results of a randomized controlled trial. $\mathrm{Br} \mathrm{J}$ Oral Maxillofac Surg. 2002;40(3):248-52.

26. Sacco R, Sacco M, Carpenedo M, Mannucci PM. Oral surgery in patients on oral anticoagulant therapy: a randomized comparison of different intensity targets. Oral Surg Oral Med Oral Pathol Oral Radiol Endod. 2007 Jul;104(1):e18-21.

27. Al-Mubarak S, Rass MA, Alsuwyed A, Alabdulaaly A, Ciancio S. Thromboembolic risk and bleeding in patients maintaining or stopping oral anticoagulant therapy during dental extraction. J Thromb Haemost JTH. 2006;4(3):689-91.

28. Sacco R, Sacco M, Carpenedo M, Moia M. Oral surgery in patients on oral anticoagulant therapy: a randomized comparison of different INR targets. J Thromb Haemost JTH. 2006;4(3):688-9.

29. Evans IL, Sayers MS, Gibbons AJ, Price G, Snooks H, \& Sugar AW. Can warfarin be continued during dental extraction? Results of a randomised controlled trial. $\mathrm{Br} J$ Oral Maxillofac Surg 2002; 40(3)248-252.

30. Sen P, Sen R. Re: Sugar AW et al. Can warfarin be continued during dental extraction? Results of a randomised controlled trial. Br J Oral Maxillofac Surg. 2003 Apr;41(2):132.

31. Bajkin BV, Popovic SL, Selakovic SDJ. Randomized, prospective trial comparing bridging therapy using low-molecular-weight heparin with maintenance of oral anticoagulation during extraction of teeth. J Oral Maxillofac Surg. 2009;67(5):990-5 32. Bailey BM, Fordyce AM. Complications of dental extractions in patients receiving warfarin anticoagulant therapy. A controlled clinical trial. Br Dent J. 1983;155(9):308-10. 33. Souto JC, Oliver A, Zuazu-Jausoro I, Vives A, Fontcuberta J. Oral surgery in anticoagulated patients without reducing the dose of oral anticoagulant: a prospective randomized study. J Oral Maxillofac Surg. 1996;54(1):27-32; discussion 323.

34. Borea G, Montebugnoli L, Capuzzi P, Magelli C. Tranexamic acid as a mouthwash in anticoagulant-treated patients undergoing oral surgery. An alternative method to discontinuing anticoagulant therapy. Oral Surg Oral Med Oral Pathol. 1993;75(1):29-31. 35. Wahl MJ, Howell J. Altering anticoagulation therapy: a survey of physicians. J Am Dent Assoc. 1996;127(5):625-6, 629-30, 633-4 passim.

36. Kamien M. Remove the tooth, but don't stop the warfarin. Aust Fam Physician 2006;35(4):233-5 